\title{
Outcome measures for assessing change over time in studies of symptomatic children with hypermobility: a systematic review
}

\author{
Muhammad Maarj ${ }^{1,2^{*}} \mathbb{0}$, Andrea Coda ${ }^{2,3}$, Louise Tofts ${ }^{1,4}$, Cylie Williams ${ }^{5}$, Derek Santos ${ }^{6}$ and Verity Pacey ${ }^{4}$
}

\begin{abstract}
Background: Generalised joint hypermobility $(\mathrm{GJH})$ is highly prevalent among children and associated with symptoms in a fifth with the condition. This study aimed to synthesise outcome measures in interventional or prospective longitudinal studies of children with GJH and associated lower limb symptoms.

Methods: Electronic searches of Medline, CINAHL and Embase databases from inception to 16th March 2020 were performed for studies of children with GJH and symptoms between 5 and 18 years reporting repeated outcome measures collected at least 4 weeks apart. Methodological quality of eligible studies were described using the Downs and Black checklist.

Results: Six studies comprising of five interventional, and one prospective observational study (total of 388 children) met the inclusion criteria. Interventional study durations were between 2 and 3 months, with up to 10 months post-intervention follow-up, while the observational study spanned 3 years. Three main constructs of pain, function and quality of life were reported as primary outcome measures using 20 different instruments. All but one measure was validated in paediatric populations, but not specifically for children with GJH and symptoms. One study assessed fatigue, reporting disabling fatigue to be associated with higher pain intensity.
\end{abstract}

Conclusions: There were no agreed sets of outcome measures used for children with GJH and symptoms. The standardisation of assessment tools across paediatric clinical trials is needed. Four constructs of pain, function, quality of life and fatigue are recommended to be included with agreed upon, validated, objective tools.

Keywords: Fatigue, Outcome measures, Paediatrics, Hypermobility, Pain, Function, Quality of life

\section{Background}

Children with generalised joint hypermobility (GJH) and associated symptoms have been described within the literature under multiple diagnostic labels which have differed over time. Generalised joint hypermobility $(\mathrm{GJH})$ describes abnormally high joint ranges of movement in multiple joints [1] with approximately one-fifth of children with GJH reporting symptoms [2,

\footnotetext{
*Correspondence: muhammad.maarj@uon.edu.au

${ }^{2}$ Department of Health Sciences, Newcastle University, Newcastle,

Australia

Full list of author information is available at the end of the article
}

3]. Currently used diagnostic labels describing children with GJH with associated symptoms include Generalised Hypermobility Spectrum Disorder (G-HSD) [4], and hypermobile Ehlers-Danlos Syndrome (hEDS), which further incorporates an extended phenotype including skin involvement, tissue fragility or a marfanoid body habitus [5]. These conditions were previously referred to as Joint Hypermobility Syndrome (JHS) or EDS-Hypermobile type, with experts previously reporting a lack of clinical distinction between the two $[6,7]$. The term "children with GJH and associated symptoms" will be used throughout this review 
to indicate any of the current or previously used terminology for this condition.

Children with GJH and associated symptoms report chronic pain [8], fatigue [9] and functional difficulties [10] that have a negative impact on their quality of life [11, 12]. Chronic joint pain is often exacerbated following physical activity [13] with lower limb pain being the most common location described [14]. Joint instability episodes and frequent soft tissue injuries have also been reported [14]. Functional difficulties reported include motor development challenges [15], muscle torque deficits and poor proprioception [16] resulting in a negative influence on school and/or social activity participation [17]. Some children with GJH also describe systemic symptoms including orthostatic intolerance, functional gastrointestinal disorders and stress incontinence $[11,14]$, with a greater number of systemic symptoms leads to worse functional disability [18]. Additional psychological symptoms may also result in poorer quality of life than typically developing children [12, 14, 19, 20].

Validated, reliable outcome measures enhance our understanding of the natural history of a condition and aid evaluation of treatment effectiveness. Despite the importance of such validated outcome measures in paediatric populations [21] there are no condition specific outcome measurement instruments for children with $\mathrm{GJH}$ and associated symptoms. Consequently, the natural history of the condition is poorly understood, and recent systematic reviews and meta-analyses have been largely inconclusive, partially due to the lack of standardised outcome measures used between studies [22-25]. Identification of outcomes reported in the literature to monitor change in children with GJH and associated symptoms informs rigorous methodology incorporating expert researcher and health professionals consensus, in conjunction with patient and family involvement, to develop a minimum core outcome set for research reporting [26]. Therefore, this study aimed to synthesise outcome measure type and use in interventional or prospective longitudinal studies of children with GJH and associated symptoms.

\section{Methods}

This systematic review was performed according to the Preferred Reporting Items for Systematic Reviews and Meta-Analyses (PRISMA) guidelines [27]. The protocol was registered on the Prospective Register of Systematic Reviews (PROSPERO) database (registration number CRD 42,018,081,835) prior to commencement of database searches.

\section{Definition of Beighton Score}

The 9-point Beighton score (BS) is a dichotomised standardised method [28, 29] widely used for assessment of GJH and associated symptoms as a clinical diagnostic tool as well as in hypermobility related research studies [30,31]. The Beighton score typically includes four or more positive finding for both upper and lower joints as follows: passive dorsiflexion of elbows, knees and fifth finger beyond $90^{\circ}$ angle; forward flexion of trunk with full extension of knees and hands resting flat on the floor; and passive opposition of each thumb to the forearm flexor surface [28].

Although the cut-off of $\geq 4 / 9$ is the most commonly used BS, however this score is largely influenced by age, gender and ethnicity [32-34]. Therefore, the new 2017 International Classification of the Ehlers-Danlos syndromes has recommended the use of age specific cut-offs of $\geq 4 / 9$ (adults older than 50 years), $\geq 5 / 9$ (pubertal children and adults 50 years or lower) or $\geq$ 6/9 (prepubertal children) for BS [5]. Since BS was originally not intended as a diagnostic tool [28] it has not been directly validated to detect GJH in adults. However BS has been found to be a valid method in assessing hypermobility in children with GJH [32].

\section{Search Strategy}

Medline (via PubMed), CINAHL and Embase databases were searched from inception to 16th March 2020 using the terms and strategy presented in Table 1. Further studies were retrieved from backward manual searches of references lists of included studies. There was no restriction imposed by publication year or language.

\section{Eligibility criteria}

Randomised controlled trials (RCTs), quasi-RCTs, longitudinal and cohort studies were included. The study populations were restricted to children and adolescents aged between 5 and 18 years, diagnosed with GJH, as defined by the authors of the studies, and associated lower limb symptoms. Given the considerable heterogeneity of cut-offs for Beighton score reported in literature its limitation as a clinical diagnostic tool [30,31], we included all relevant studies that assessed children with GIH and associated symptoms. Included studies were required to describe outcome measures utilised at least 4 weeks apart in order to identify change over time.

Studies focusing on upper limb only outcome measures, or studies including children with other hereditary connective tissue disorders or syndromic conditions associated with $\mathrm{GJH}$, were not included. 
Table 1 Search terms and search strategy documentation for PubMed ${ }^{a}$

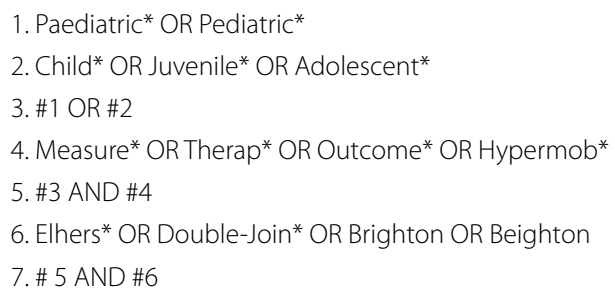

${ }^{\text {a }}$ This search strategy was modified for CINAHL and Embase databases

\section{Study selection}

Titles, abstracts and full-text article screening was performed independently by two authors (MM and $\mathrm{AC}$ ) against the inclusion/exclusion criteria. Any discrepancies were resolved either by discussion between the two reviewers or by a third author (DS) until consensus was reached.

\section{Data extraction}

Two reviewers (MM and CW) independently extracted relevant data from included full text articles. Data extraction was performed on a standardised template and included: the primary author of the study, year of publication, country, study design, participant demographics (sample size, gender and age), intervention characteristics (type, duration and follow-up) where applicable, and outcome measures used to assess change. Any unresolved disagreements were mediated by the remaining authors (AC, LT, DS and VP).

\section{Risk of bias assessment}

The methodological quality of all eligible studies was reviewed independently by two authors (MM and DS) using the Downs and Black checklist. Any disagreements were discussed until a consensus was reached or resolved by a third author (AC). The Downs and Black checklist [35] is a validated methodological quality assessment tool covering 5 domains of reporting, external quality, internal validity (bias), internal validity confounding or selection bias, and statistical power [36].

\section{Data analysis}

Descriptive statistics were used to characterise the included studies participant population, duration and intervention. Outcome measures used were categorised into patient- or parent-reported (PRO) or clinicianreported (CRO) outcomes, and the broad constructs which were being assessed. The frequency of individual outcome measures used to assess each construct was then tallied. A narrative synthesis of the outcome measures used across study type and participant age was performed, including presentation of the baseline scores on measures. To provide a description of the change over time, the mean change, and variance in this, was also presented. Where $95 \%$ CIs were not presented to represent the variance in change, they were calculated.

\section{Results}

Selection strategy and methodological appraisal

From a total of 1136 articles identified through the searches, 57 articles were deemed eligible for full-text screening with six studies eligible to be included in this review (Fig. 1). Five interventional studies were identified, these were four RCTs and one pre-post cohort study. The sixth was a prospective observational study. All included studies were published during the last ten years.

The methodological quality of the six studies was described in Table 2. Main limitations of the studies included poor description of principal confounders, lack of participant blinding, not reporting adverse events related to intervention(s), and not minimising bias for data collection. The strength of included studies were clearly described main outcomes, recruitment of participants from the same target population as well as the use of validated and reliable outcome measures appropriate for the general paediatric population. While all interventional studies clearly described the trial and control interventions, only one study blinded participants to the interventions while the other four studies demonstrated blinding of assessors to the group allocation of intervention or controls.

\section{Characteristics of the eligible studies}

The main characteristics of included studies are summarised in Table 3. There were 388 participants in total from the six studies. Overall, studies included primarily female participants, and ranged in duration from 2 months to 3 years. Interventions included either exercise therapy alone $(n=3)$ or combined with orthotics $(n=1)$ or multidisciplinary care $(n=2)$. All participants were recruited from children's hospital clinics. 


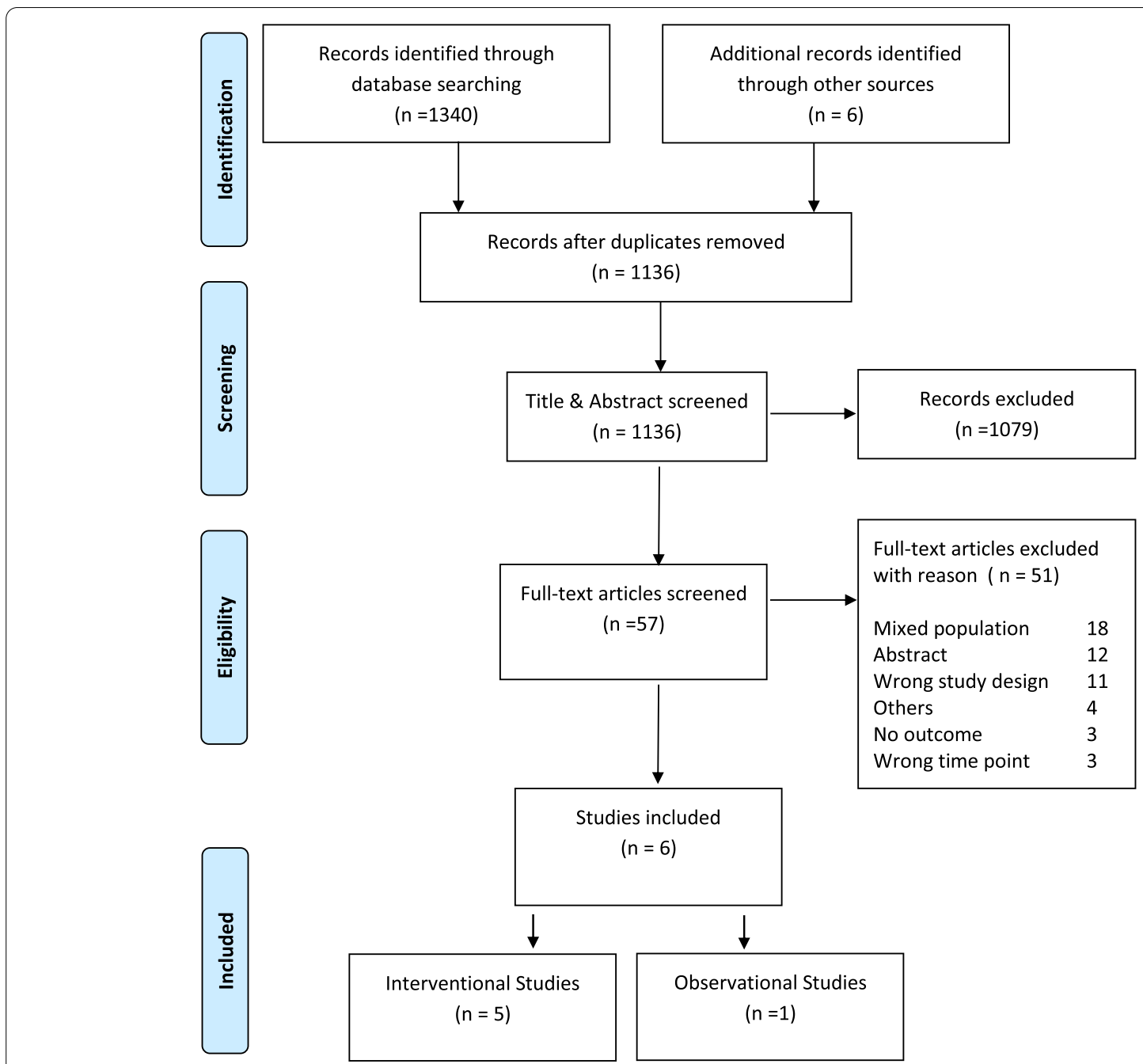

Fig. 1 Flow diagram of the study

\section{Outcome measures}

Table 4 provides descriptions of the outcome measures and instruments used in the studies where the change in these measures over time was able to be collected or provided by the authors. There were 20 distinct outcome instruments measuring the four constructs of pain [39-42, 44], function [39-44], quality of life [39-42, 44] and fatigue [44] which included 15 PROs (7 patientreported and 8 parent-reported) and 4 CROs. All PRO instruments except one (PGIC: Patient's Global Impression of Change) [42] have been validated for use in the paediatric population. Pain was the most common construct measured, using 4 different PROs [39, 41-44], the patient-reported Visual Analogue Scale (VAS) [39, 41, 42, 44], parent-reported VAS [39, 41], Numerical Rating Scale (NRS) [43], and the Wong-Baker Faces Pain Scale (WBFPS) [39].
When considering all the PROs used, the patientreported VAS [39, 41, 42, 44], Childhood Health Assessment Questionnaire (CHAQ) [39, 41, 42, 44] and parent-reported VAS $[39,41]$ were the only PRO measures used in more than one study.

Function was assessed with a total of nine different assessment tools. Five PROs were used to assess function including the CHAQ [39, 41, 42], Pediatric Outcomes Data Collection Instrument (PODCI) [40], and the Bath Adolescent Pain questionnaire (BAPQ 61) [43]. The Bath Adolescent Pain Parent Impact Questionnaire (BAP-PIQ) was also used to assess the impact of the child's condition on the parents daily function [43], and the Adolescent Physical Activity Recall Questionnaire (APARQ) scale to assess a child's physical activity [44]. The 4 CROs used to assess function included the 6 min walking test to assess walking endurance [44], the ability to climb stairs in a set time [42], the Movement Assessment Battery 
Table 2 Assessment of methodological quality of eligible studies using Downs \& Black checklist (Downs and Black 1998)a

\begin{tabular}{|c|c|c|c|c|c|c|c|}
\hline Items & Criteria & Bale (2019) & Hsieh (2018) & $\begin{array}{l}\text { Revivo } \\
(2018)\end{array}$ & Pacey (2013) & Kemp (2010) & Scheper (2017) \\
\hline \multicolumn{8}{|c|}{ REPORTING } \\
\hline 1 & Study hypothesis/aim/objective clearly described & 1 & 1 & 1 & 1 & 1 & 0 \\
\hline 2 & Main outcomes in Introduction or Methods section & 1 & 1 & 1 & 1 & 1 & 1 \\
\hline 3 & Patient characteristics clearly described & 1 & 1 & 1 & 1 & 1 & 1 \\
\hline 4 & $\begin{array}{l}\text { Relevant interventions including controls clearly } \\
\text { described }\end{array}$ & 1 & 1 & 1 & 1 & 1 & NA \\
\hline 5 & $\begin{array}{l}\text { Distributions of principal confounders clearly } \\
\text { described }\end{array}$ & 0 & 0 & 1 & 0 & 0 & 2 \\
\hline 6 & Main findings (including outcomes) clearly described & 1 & 1 & 1 & 1 & 1 & 1 \\
\hline 7 & $\begin{array}{l}\text { Estimates of random variability in data for the main } \\
\text { outcomes provided }\end{array}$ & 1 & 1 & 1 & 1 & 1 & 1 \\
\hline 8 & $\begin{array}{l}\text { All important adverse events related to intervention(s) } \\
\text { reported }\end{array}$ & 0 & 0 & 0 & 1 & 0 & NA \\
\hline 9 & Patient characteristics lost to follow-up described & 1 & 1 & 1 & 1 & 0 & 0 \\
\hline 10 & Actual probability values for main outcomes reported & 1 & 1 & 1 & 1 & 1 & 1 \\
\hline \multicolumn{8}{|c|}{ EXTERNAL VALIDITY } \\
\hline 11 & $\begin{array}{l}\text { Subjects asked to participate were representative of } \\
\text { target populations }\end{array}$ & 1 & 1 & 1 & 1 & 1 & 1 \\
\hline 12 & $\begin{array}{l}\text { Subjects prepared to participate were representative } \\
\text { of target populations }\end{array}$ & 1 & 1 & 1 & 1 & 1 & 1 \\
\hline 13 & $\begin{array}{l}\text { Treatment facilities and delivery were representative of } \\
\text { target populations }\end{array}$ & 1 & 1 & 1 & 1 & 1 & 1 \\
\hline \multicolumn{8}{|c|}{ INTERNAL VALIDITY - bias } \\
\hline 14 & $\begin{array}{l}\text { Study participants blinded to intervention adminis- } \\
\text { tered }\end{array}$ & 0 & 0 & 0 & 1 & 0 & NA \\
\hline 15 & $\begin{array}{l}\text { Investigators blinded to assessment of main interven- } \\
\text { tion outcomes }\end{array}$ & 1 & 1 & 0 & 1 & 1 & NA \\
\hline 16 & Any data dredging was made clear at onset of study & 0 & 0 & 1 & 1 & 1 & 0 \\
\hline 17 & $\begin{array}{l}\text { Analyses adjust for different lengths of follow-up of } \\
\text { participants }\end{array}$ & 1 & 0 & 1 & 1 & 0 & 1 \\
\hline 18 & $\begin{array}{l}\text { Statistical tests to assess the main outcomes were } \\
\text { appropriate }\end{array}$ & 1 & 1 & 1 & 1 & 1 & 1 \\
\hline 19 & Reliability of compliance with intervention(s) & 1 & 1 & 1 & 1 & 0 & NA \\
\hline 20 & $\begin{array}{l}\text { Main outcome measures used accurate in terms of } \\
\text { validity and reliability. }\end{array}$ & 1 & 1 & 1 & 1 & 1 & 1 \\
\hline \multicolumn{8}{|c|}{ INTERNAL VALIDITY - confounding (selection bias) } \\
\hline 21 & $\begin{array}{l}\text { All participants were recruited from the same target } \\
\text { population }\end{array}$ & 1 & 1 & 1 & 1 & 1 & 1 \\
\hline 22 & $\begin{array}{l}\text { All participants were recruited over the same period } \\
\text { of time }\end{array}$ & 1 & 1 & 0 & 1 & 1 & 1 \\
\hline 23 & Participants were randomised to intervention group(s) & 1 & 1 & 0 & 1 & 1 & NA \\
\hline 24 & $\begin{array}{l}\text { Randomised intervention assignment was concealed } \\
\text { from both participants and investigators }\end{array}$ & 0 & 0 & 0 & 1 & 0 & NA \\
\hline 25 & Adequate adjustment for confounding & 0 & 0 & 0 & 0 & 0 & 1 \\
\hline 26 & Lost to follow-up considered & 1 & 0 & 1 & 1 & 0 & 0 \\
\hline 27 & $\begin{array}{l}\text { Statistical power- clinical meaningful effect or power } \\
\text { calculation reported }{ }^{\text {b }}\end{array}$ & $1^{\sim}$ & 1 & 1 & 1 & 1 & 1 \\
\hline
\end{tabular}

$\sim$ Power calculation reported but not clinically meaningful

a The scoring given for each criteria was 1 point for 'Yes' or 0 point for 'No' except question 5 which is scored as 2 for 'Yes', 1 for partially or 0 for 'No' related to the distribution of principle confounders [35]. For observational study NA=Not applicable.

b Only one point was awarded to an interventional study powered to detect a meaningful clinical effect $[37,38]$ 


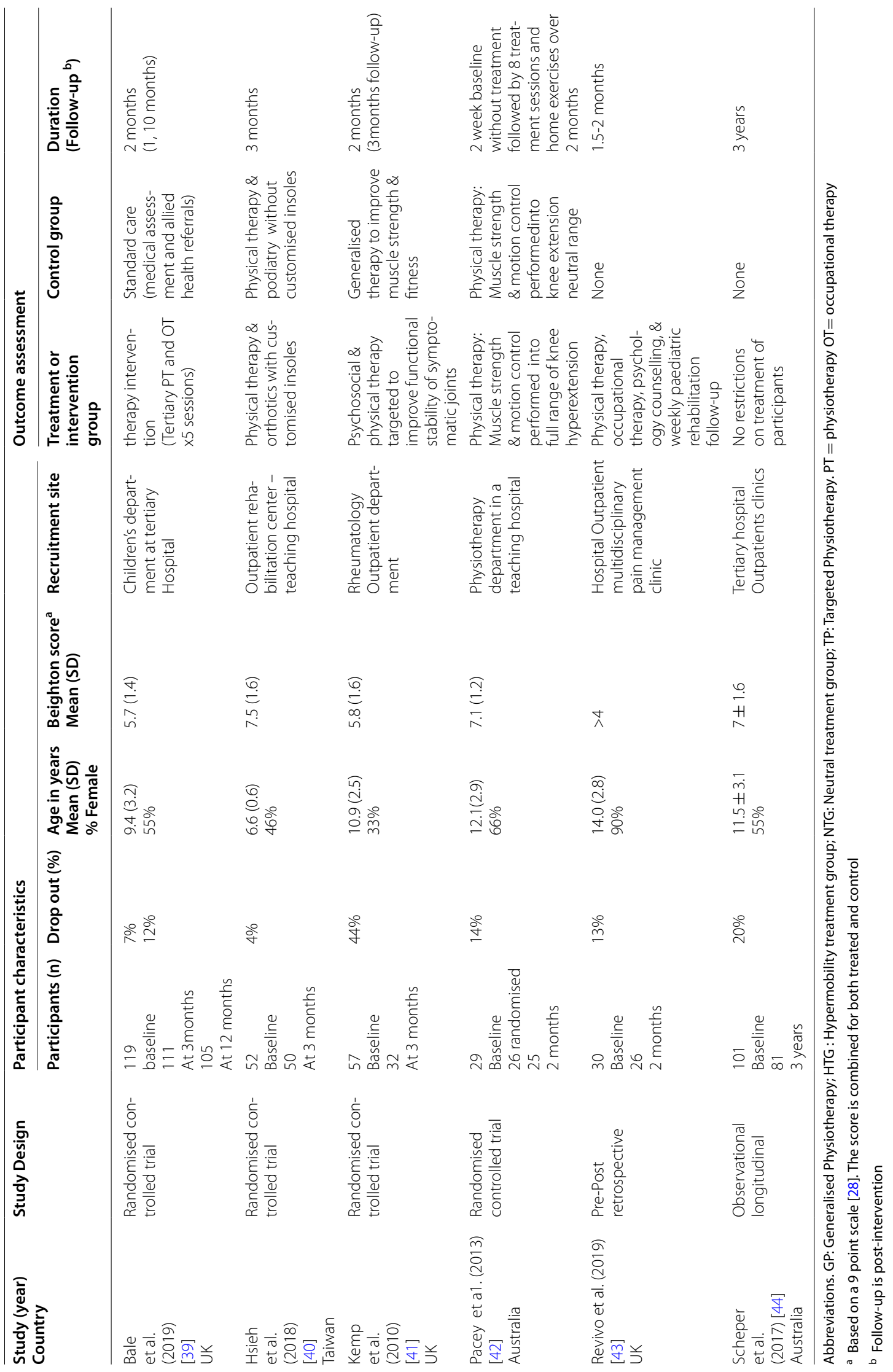


Table 4 Outcome measures categorised according to pain, function and quality of life

\begin{tabular}{|c|c|c|c|c|c|c|}
\hline \multicolumn{3}{|l|}{ Outcome measures } & \multirow[t]{2}{*}{ Follow-up Timeframe ${ }^{d}$} & \multirow{2}{*}{$\begin{array}{l}\text { Baseline } \\
\text { Mean (SD) }\end{array}$} & \multirow{2}{*}{$\begin{array}{l}\text { Mean change } \\
\text { in outcome at } \\
\text { follow-up }{ }^{a}\end{array}$} & \multirow[t]{2}{*}{$95 \% \mathrm{Cl}$} \\
\hline Scale & Test details & Type & & & & \\
\hline \multicolumn{7}{|l|}{ PAIN (Intensity) } \\
\hline \multirow[t]{6}{*}{$\begin{array}{l}\text { VAS }[45,46] \\
\text { (Visual Analogue scale) }\end{array}$} & \multirow{6}{*}{$\begin{array}{l}0-100 \\
0=\text { no pain } \\
100=\text { worst pain }\end{array}$} & \multirow[t]{6}{*}{$\mathrm{PRO}$} & \multirow[t]{3}{*}{2 months [42] } & $\begin{array}{l}\text { Neutral treatment group: } \\
40.0 \text { (16.6) }\end{array}$ & -19.9 & NR \\
\hline & & & & $\begin{array}{l}\text { Hypermobility treatment } \\
\text { group: } 38.6 \text { (16.9) }\end{array}$ & -9.19 & NR \\
\hline & & & & $\begin{array}{l}\text { Combined groups: } 39.4 \\
(14.2)\end{array}$ & -14.5 & $-5.2,-23.8$ \\
\hline & & & \multirow[t]{3}{*}{$\begin{array}{l}5 \text { months } \\
\text { [41] }\end{array}$} & $\begin{array}{l}\text { Targeted Physiotherapy: } \\
55.5 \text { (21.3) }\end{array}$ & -21.2 & $-38,-4.5$ \\
\hline & & & & $\begin{array}{l}\text { General Physiotherapy: } \\
62.1(24.1)\end{array}$ & -30.6 & $-50.16,-11.0$ \\
\hline & & & & $\begin{array}{l}\text { Combined groups: } 57.6 \\
\text { (20.1) }\end{array}$ & -25.8 & $-38.5,-13.1$ \\
\hline \multirow{2}{*}{$\begin{array}{l}\text { WBFPS }[47,48] \\
\text { (Wong-baker faces pain } \\
\text { scale) }\end{array}$} & $0-5$ & \multirow[t]{2}{*}{$\mathrm{PRO}$} & \multirow{2}{*}{$\begin{array}{l}12 \text { months } \\
{[39]}\end{array}$} & Intervention: 2.2 (1.4) & -1.6 & $-2.1,-1.1$ \\
\hline & $\begin{array}{l}0=\text { no pain } \\
5=\text { worst pain }\end{array}$ & & & Control: 2.5 (1.6) & -1.6 & $-2.0,-1.2$ \\
\hline \multicolumn{7}{|l|}{ PAIN (Intensity) } \\
\hline \multirow{5}{*}{$\begin{array}{l}\text { VAS-P [49] } \\
\text { (Visual Analogue scale- } \\
\text { Parental) }\end{array}$} & \multirow{5}{*}{$\begin{array}{l}0-100 \\
0=\text { no pain } \\
100=\text { worst pain }\end{array}$} & \multirow[t]{5}{*}{$\mathrm{PRO}^{\mathrm{b}}$} & \multirow[t]{3}{*}{$\begin{array}{l}5 \text { months } \\
{[41]}\end{array}$} & $\begin{array}{l}\text { Targeted Physiotherapy: } \\
45.1 \text { (23.0) }\end{array}$ & -21.6 & $-33.2,-10.0$ \\
\hline & & & & $\begin{array}{l}\text { General Physiotherapy: } \\
48.4(22.9)\end{array}$ & -12 & $-23.3,0.9$ \\
\hline & & & & $\begin{array}{l}\text { Combined groups: } \\
46.7(22.7)\end{array}$ & -17.2 & $-25.3,-9.1$ \\
\hline & & & \multirow{2}{*}{$\begin{array}{l}12 \text { months } \\
{[39]}\end{array}$} & Intervention: 33.8 (24.8) & -6.8 & $-14.3,0.7$ \\
\hline & & & & Control: 40.6 (27.5) & -7.3 & $-15.4,0.8$ \\
\hline \multicolumn{7}{|l|}{ FUNCTION } \\
\hline \multirow{8}{*}{$\begin{array}{l}\text { CHAQ [50] (Childhood } \\
\text { Health Assessment } \\
\text { Questionnaire) }\end{array}$} & \multirow{8}{*}{$\begin{array}{l}0-3 \\
0=\text { Without any difficulty } \\
1=\text { With some difficulty } \\
2=\text { With much difficulty } \\
3=\text { Unable to do }\end{array}$} & \multirow[t]{8}{*}{$\mathrm{PRO}^{\mathrm{b}}$} & \multirow[t]{3}{*}{$\begin{array}{l}2 \text { months } \\
{[42]}\end{array}$} & $\begin{array}{l}\text { Neutral treatment group: } \\
-0.13(0.44)\end{array}$ & 0.12 & NR \\
\hline & & & & $\begin{array}{l}\text { Hypermobility treatment } \\
\text { group: } \\
0.04(0.71)\end{array}$ & 0.02 & $N R$ \\
\hline & & & & $\begin{array}{l}\text { Combined groups: }-0.5 \\
(0.6)\end{array}$ & 0.07 & $-0.1,0.2$ \\
\hline & & & \multirow[t]{3}{*}{$\begin{array}{l}5 \text { months } \\
{[41]}\end{array}$} & $\begin{array}{l}\text { Targeted Physiotherapy: } \\
0.62(0.65)\end{array}$ & -0.15 & $-0.3,-0.02$ \\
\hline & & & & $\begin{array}{l}\text { General Physiotherapy: } \\
0.76(0.68)\end{array}$ & -0.16( & $-0.4,0.1$ \\
\hline & & & & $\begin{array}{l}\text { Combined groups: } 0.69 \\
(0.66)\end{array}$ & -0.15 & $-0.3,-0.02$ \\
\hline & & & \multirow{2}{*}{$\begin{array}{l}12 \text { months } \\
{[39]}\end{array}$} & Intervention: 0.84 (0.62) & 0.04 & $0.1,0.2$ \\
\hline & & & & Control: 0.86 (0.72) & -0.02 & $-0.12,0.08$ \\
\hline \multirow{5}{*}{$\begin{array}{l}\text { Dynamometry [51] } \\
\text { Measurement of strength }\end{array}$} & \multirow{2}{*}{$\begin{array}{l}\text { Grip strength. Units: } \\
\text { kilopascals }\end{array}$} & \multirow[t]{5}{*}{$\mathrm{CRO}$} & 12 months & Intervention: 57.0 (25.0) & 4.7 & $0.1,9.3$ \\
\hline & & & & Control: 59.4 (31.7) & 7.3 & $2.9,11.7$ \\
\hline & $\begin{array}{l}\text { Knee flexor and extensor } \\
\text { strength. Units: Newtons }\end{array}$ & & $\begin{array}{l}2 \text { months } \\
\text { [42] }\end{array}$ & $\begin{array}{l}\text { Neutral treatment group: } \\
4.0(1.7)\end{array}$ & 0.88 & NR \\
\hline & & & & $\begin{array}{l}\text { Hypermobility treatment } \\
\text { group: } 4.4 \text { (2.4) }\end{array}$ & 1.21 & NR \\
\hline & & & & $\begin{array}{l}\text { Combined groups: } 4.2 \\
(2.0)\end{array}$ & 1.1 & $0.4,1.7$ \\
\hline
\end{tabular}


Table 4 (continued)

\begin{tabular}{|c|c|c|c|c|c|c|}
\hline \multicolumn{3}{|l|}{ Outcome measures } & \multirow[t]{2}{*}{ Follow-up Timeframe ${ }^{d}$} & \multirow{2}{*}{$\begin{array}{l}\text { Baseline } \\
\text { Mean (SD) }\end{array}$} & \multirow{2}{*}{$\begin{array}{l}\text { Mean change } \\
\text { in outcome at } \\
\text { follow-up }^{\text {a }}\end{array}$} & \multirow[t]{2}{*}{$95 \% \mathrm{Cl}$} \\
\hline Scale & Test details & Type & & & & \\
\hline \multicolumn{7}{|l|}{ FUNCTION } \\
\hline \multirow{2}{*}{$\begin{array}{l}\text { M-ABC2 [52] } \\
\text { (Movement Assessment } \\
\text { Battery for Children, 2nd } \\
\text { Edition) }\end{array}$} & \multirow{2}{*}{$\begin{array}{l}\text { Measures coordination } \\
\text { Raw scores converted } \\
\text { to centiles }(0-100) \text { with } \\
\text { higher centiles indicat- } \\
\text { ing better performance } \\
\text { compared to peers }\end{array}$} & \multirow[t]{2}{*}{$\mathrm{CRO}$} & \multirow{2}{*}{$\begin{array}{l}12 \text { months } \\
{[39]}\end{array}$} & Intervention: 33.4 (26.7) & 3.8 & $-1.7,9.3$ \\
\hline & & & & Control: 35.6 (30.1) & 10.8 & $5.4,16.2$ \\
\hline \multirow[t]{2}{*}{$\begin{array}{l}\text { PODCI }[53,54] \\
\text { (Pediatric Outcomes Data } \\
\text { Collection Instrument) }\end{array}$} & \multirow[t]{2}{*}{$\begin{array}{l}0-100 \\
100=\text { highest function- } \\
\text { ing }\end{array}$} & \multirow[t]{2}{*}{$\mathrm{PRO}^{\mathrm{b}}$} & \multirow[t]{2}{*}{$\begin{array}{l}3 \text { months } \\
{[40]}\end{array}$} & $\begin{array}{l}\text { Transfer and basic mobil- } \\
\text { ity domain } \\
\text { Intervention: } 82.1 \text { (14.8) }\end{array}$ & 11.8 & $0.30,1.43$ \\
\hline & & & & Control: 94.2 (14.8) & 1.2 & $-0.46,0.62$ \\
\hline \multirow[t]{2}{*}{$\begin{array}{l}6 \mathrm{MWT}[55] \\
\text { (6 min walk test) }\end{array}$} & \multirow{2}{*}{$\begin{array}{l}\text { Maximum distance } \\
\text { walked in } 6 \text { minutes } \\
\text { (meters/leg length) }\end{array}$} & \multirow[t]{2}{*}{$\mathrm{CRO}$} & \multirow[t]{2}{*}{$\begin{array}{l}36 \text { months } \\
{[44]}\end{array}$} & $\begin{array}{l}\text { Able/moderate: } 7.3 \\
(1.6)^{c}\end{array}$ & -1.5 & $-1.3,-4.5$ \\
\hline & & & & Severe: 5.3 (1.6) & -2.3 & $-2.4,-2.7$ \\
\hline \multirow{3}{*}{$\begin{array}{l}\text { No. of flights of stairs } \\
\text { climbed in } 2 \mathrm{~min} \\
{[56]}\end{array}$} & \multirow[t]{3}{*}{$\begin{array}{l}\text { Assesses functional abil- } \\
\text { ity in stairclimbing }\end{array}$} & \multirow[t]{3}{*}{$\mathrm{CRO}$} & \multirow[t]{3}{*}{$\begin{array}{l}2 \text { months } \\
{[42]}\end{array}$} & $\begin{array}{l}\text { Neutral treatment group: } \\
16.3(5.0)\end{array}$ & 3.8 & NR \\
\hline & & & & $\begin{array}{l}\text { Hypermobility treatment } \\
\text { group: } 20.9(6.7)\end{array}$ & -0.33 & NR \\
\hline & & & & $\begin{array}{l}\text { Combined groups: } 18.6 \\
(5.7)\end{array}$ & 1.7 & $-0.5,3.9$ \\
\hline \multicolumn{7}{|l|}{ Quality of Life } \\
\hline \multirow{2}{*}{$\begin{array}{l}\text { CHU9D [57] } \\
\text { (Child Health Utility 9D) }\end{array}$} & \multirow{2}{*}{$\begin{array}{l}0-4 \\
\text { higher scores indicate } \\
\text { poorer HRQoL }\end{array}$} & \multirow[t]{2}{*}{ PRO } & \multirow{2}{*}{$\begin{array}{l}12 \text { months } \\
{[39]}\end{array}$} & Intervention: 0.85 (0.10) & $0.02(0.09)$ & $-0.004,0.04$ \\
\hline & & & & Control: 0.85 (0.12) & $0.00(0.12)$ & $-0.03,0.03$ \\
\hline \multirow{8}{*}{$\begin{array}{l}\text { CHQ-PF50 [58] } \\
\text { (Child Health Question- } \\
\text { naire) }\end{array}$} & \multirow{8}{*}{$\begin{array}{l}0-100 \\
0=\text { worst QoL } \\
100=\text { Best QoL }\end{array}$} & $\mathrm{PRO}^{\mathrm{b}}$ & 2 months [42] & Physical summary score & & \\
\hline & & & & $\begin{array}{l}\text { Neutral treatment group: } \\
32.0(11.9)\end{array}$ & 10.1 & NR \\
\hline & & & & $\begin{array}{l}\text { Hypermobility treatment } \\
\text { group: } 41.6 \text { (15.0) }\end{array}$ & 2.3 & NR \\
\hline & & & & $\begin{array}{l}\text { Combined groups: } 38.0 \\
\text { (12.6) }\end{array}$ & 5.3 & $1.7,8.9$ \\
\hline & & & & Psychological summary sco & & \\
\hline & & & & $\begin{array}{l}\text { Neutral treatment group: } \\
46.4(12.3)\end{array}$ & -0.9 & NR \\
\hline & & & & $\begin{array}{l}\text { Hypermobility treatment } \\
\text { group: } 46.3 \text { (9.0) }\end{array}$ & 8.1 & NR \\
\hline & & & & $\begin{array}{l}\text { Combined groups: } 48 \\
(10.3)\end{array}$ & 2.7 & $-0.3,5.8$ \\
\hline Quality of Life & & & & & & \\
\hline $\begin{array}{l}\text { PGIC }[59,60] \\
\text { (Patient global impres- }\end{array}$ & $\begin{array}{l}1-7 \\
1=\text { very much improved }\end{array}$ & $\mathrm{PRO}$ & $\begin{array}{l}2 \text { months } \\
{[42]}\end{array}$ & $\begin{array}{l}\text { Neutral treatment group: } \\
0.3(1.1)\end{array}$ & 1.4 & NR \\
\hline sion of change) & $\begin{array}{l}7=\text { very much } \\
\text { worse }\end{array}$ & & & $\begin{array}{l}\text { Hypermobility treatment } \\
\text { group: } 0.2(0.9)\end{array}$ & 1.6 & NR \\
\hline & & & & $\begin{array}{l}\text { Combined groups: } 0.2 \\
(1.0)\end{array}$ & 1.5 & $1.0,2.0$ \\
\hline PODCI [53] (Pediatric & $0-100$ & $\mathrm{PRO}^{\mathrm{b}}$ & 3 months & 1. Pain/comfort: & & \\
\hline $\begin{array}{l}\text { Outcomes Data Collec- } \\
\text { tion Instrument) }\end{array}$ & $\begin{array}{l}\text { Higher score means } \\
\text { higher health-related }\end{array}$ & & & $\begin{array}{l}\text { Intervention: } \\
83.9(16.2)\end{array}$ & 4.9 & $-0.22,0.87$ \\
\hline $\begin{array}{l}\text { Paln comfort } \\
\text { Happiness }\end{array}$ & & & & Control: 84.4 (17) & -1.2 & $-0.61,0.47$ \\
\hline & & & & 2. Happiness: & & \\
\hline & & & & Intervention: 79.5 (18.7) & -0.2 & $-0.55,0.53$ \\
\hline & & & & Control: 80.7 (15.9) & -0.9 & $-0.60,0.48$ \\
\hline
\end{tabular}


Table 4 (continued)

\begin{tabular}{|c|c|c|c|c|c|c|}
\hline \multicolumn{3}{|l|}{ Outcome measures } & \multirow[t]{2}{*}{ Follow-up Timeframe ${ }^{d}$} & \multirow{2}{*}{$\begin{array}{l}\text { Baseline } \\
\text { Mean (SD) }\end{array}$} & \multirow{2}{*}{$\begin{array}{l}\text { Mean change } \\
\text { in outcome at } \\
\text { follow-up }^{\text {a }}\end{array}$} & \multirow[t]{2}{*}{$95 \% \mathrm{Cl}$} \\
\hline Scale & Test details & Type & & & & \\
\hline \multirow{6}{*}{$\begin{array}{l}\text { PedsQL parent proxy- } \\
\text { reported format [61] and } \\
\text { [62] (Pediatric Quality of } \\
\text { Life Inventory-Generic } \\
\text { Core Scale) }\end{array}$} & \multirow{6}{*}{$\begin{array}{l}0-100 \\
\text { Lower score indicates } \\
\text { lower quality of life }\end{array}$} & \multirow[t]{6}{*}{$\mathrm{PRO}^{\mathrm{b}}$} & \multirow{6}{*}{$\begin{array}{l}3 \text { months } \\
{[40]}\end{array}$} & \multicolumn{3}{|l|}{ 1. Physical } \\
\hline & & & & $\begin{array}{l}\text { Intervention: } \\
62.3(19.9)\end{array}$ & 3.9 & $-0.35,0.73$ \\
\hline & & & & Control: 79.2 (20.1) & -8.1 & $-0.95,0.14$ \\
\hline & & & & \multicolumn{3}{|l|}{ 2. Psychosocial } \\
\hline & & & & $\begin{array}{l}\text { Intervention: } \\
65.6(16.3)\end{array}$ & 0.9 & $-0.49,0.59$ \\
\hline & & & & Control: 73.8 (18.8) & 0.3 & $-0.52,0.56$ \\
\hline \multicolumn{7}{|l|}{ Quality of Life } \\
\hline \multirow[t]{3}{*}{$\begin{array}{l}\text { Global-VAS (parent's } \\
\text { global assessment) }\end{array}$} & \multirow{3}{*}{$\begin{array}{l}0-100 \\
0=\text { no impact of hyper- } \\
\text { mobility } \\
100=\text { high impact of } \\
\text { hypermobility }\end{array}$} & \multirow[t]{3}{*}{$\mathrm{PRO}^{\mathrm{b}}$} & \multirow[t]{3}{*}{$\begin{array}{l}5 \text { months } \\
{[41]}\end{array}$} & $\begin{array}{l}\text { Targeted Physiotherapy: } \\
36.1 \text { (26.4) } \\
\text { General }\end{array}$ & -17.6 & $-31.1,-4.1$ \\
\hline & & & & $\begin{array}{l}\text { Physiotherapy: } \\
37.2(25.3)\end{array}$ & 3.7 & $-7.8,15.3$ \\
\hline & & & & $\begin{array}{l}\text { Combined groups: } 36.6 \\
(25.7) n=32\end{array}$ & -7.6 & $-17.2,-2.0$ \\
\hline \multicolumn{7}{|c|}{$\begin{array}{l}\text { Abbreviations. 95\% Cl: } 95 \% \text { Confidence Interval; CRO: Clinician-reported outcome; GP: Generalised Physiotherapy; HRQOL=health-related quality of life; QoL: quality } \\
\text { of life; PRO: Patient-reported outcome }\end{array}$} \\
\hline \multicolumn{7}{|c|}{ a difference in change score from baseline (outcome-baseline) } \\
\hline \multicolumn{7}{|c|}{${ }^{\mathrm{b}}$ Indicates Parent reported outcomes } \\
\hline \multicolumn{7}{|c|}{$\begin{array}{l}\text { ' Data calculated by primary author to demonstrate the difference between children of different severity and supplied to the authorship team upon request. This was } \\
\text { only able to be provided for 6MWT, not the other variables }\end{array}$} \\
\hline \multicolumn{7}{|c|}{${ }^{\mathrm{d}}$ Time points are when outcome measurements are reported } \\
\hline \multicolumn{7}{|c|}{ Authors converted the faces pain scale to a $0-100$ scale to combine with VAS data } \\
\hline
\end{tabular}

for Children, 2nd Edition (M-ABC2) [39] to assess gross motor skills, and muscle strength [39, 42]. Strength was measured in two studies, however they each assessed different muscle groups $[39,42]$.

Quality of life was described using the three different patient-reported outcome scales; Child Health Utility 9D (CHU9D) [39], PGIC [42] and Pediatric Quality of life questionnaire (PedsQL) [44]. The change in the child's quality of life reported by parents was measured using PODCI [40], Child Health Questionnaire (CHQ-PF50) [42], PedsQL parent proxy-reported format [40], and Global-VAS (parent's global assessment) [41]. Only one study measured fatigue, using the PedsQL- Multi-dimensional Fatigue Scale [44].

\section{Discussion}

There was significant heterogeneity in the use of instruments across studies included within this systematic review. Multiple studies measured pain intensity, function and quality of life constructs; however fatigue was measured in only one study, which found it to be an independent predictor of functional deterioration. All measures used demonstrated change over time.

The identified PRO measures used similar item sets without taking into account lifestyle or severity of the condition. This limits their translational capabilities into clinical practice. Despite the advantage of assessing the same outcome repeatedly in a clinical trial for research, measuring changes in symptoms tailored to the child's individual presentation may be more beneficial to inform clinical decisions [63]. Children with GJH and associated symptoms commonly describe variable symptoms depending on their lifestyles, environmental condition or individual characteristics [64]. The use of PROs with more inclusive questions that capture all relevant domains to an individual and their specific condition may provide a more useful alternative to better assist clinicians translate evidence into practice. Furthermore, the use of measures specifically validated for children with GJH and associated symptoms, would provide a clearer understanding of the natural change in symptomatology of children with GJH and associated symptoms, and more robust 
evidence for the effectiveness of interventions in this patient population.

Therapy aims to improve quality of life and reduce disability in children with GJH and associated symptoms [65]. It is unknown if generic outcome measures alone would enable reporting with adequate validity and sensitivity [66, 67]. In this present review, the majority of studies administered multiple instruments, combining both PRO and CRO scales. Further evaluation with qualitative methodology may provide valuable insight into the priorities and needs of children with $\mathrm{GJH}$ and associated symptoms, and their caregivers. This may refine the constructs and specific outcome measures used in future research and clinical practice.

Studies of intervention effectiveness and/or change with time in well described and defined populations with symptomatic generalised hypermobility using well validated robust measures that can be used in both the research and clinical contexts will support easier interpretation and comparison across both contexts. Each individual study provides valuable additional original information, but analysis of multiple studies will provide a higher level of evidence in the future This allows for comparisons between settings, interventions and patient groups to get a broader understanding of the measures used and provide meaningful informed assessment of therapies. Lack of standardisation, together with the limited number of interventional or prospective cohort studies, has hampered quantitative synthesis of efficacy of interventions using meta-analysis in previous systematic reviews $(23,24)$. In other paediatric rheumatological health conditions, such as Juvenile Idiopathic Arthritis (JIA), established and revised core sets of outcomes determined through expert health professional consensus $[68,69]$ have been used. In line with the findings of our review, the JIA international workgroup prioritised pain, function and quality of life (overall wellbeing) as mandatory domains for research. In addition, fatigue prioritised by patient/parents was considered an important construct outcome measure for inclusion in the most recent update [69].

There is a substantial impact of fatigue on quality of life of children with GJH and associated symptoms [12, 14, 18, 19, 70]. The most poorly functioning children diagnosed with hypermobility and associated symptoms experience worse fatigue and higher pain intensity than their peers [44]. No single assessment instrument has been identified to measure the severity of fatigue and its impact on wellbeing in this population group. Given the significance of fatigue, strong consideration of fatigue measurement is recommended within a core set of outcome measures.

Studies have also reported children and parents describing systemic symptoms such as gastrointestinal involvement and stress incontinence associated with poorer quality of life relating to hypermobility [14, $71,72]$. Outcome measures measure that identify the impact of different systemic symptoms on child function and quality of life may also be useful to guide clinical management and assess the efficacy of interventions in this population.

This review was strengthened through the registration of a protocol, adherence to established PRISMA guidelines, and appraisal of methodological quality using a tool with substantial inter-rater reliability [73], and one that highlighted for use in assessing the quality of non-randomised controlled studies [74]. We acknowledge a number of limitations to this review. The research strategy used within this review only identified studies published in English despite no language restrictions placed on eligibility criteria. This study also focused on outcome measures for children with GJH and associated lower limb symptoms and did not assess outcome measures relevant to children's other symptoms affecting the upper limb and spine, or other multisystemic features. While limiting the review, lower limb symptoms were chosen as they are consistently reported most frequently in this population [75]. Additionally, it was not the aim of the review to assess the validity or reliability of the included measures in the paediatric or condition-specific population.

We are also not able to comment on the association between degree of joint hypermobility and the outcomes of pain, fatigue, quality of life and function since there is no available clinical diagnostic markers for hypermobility disorders or tools to assess such relationship. The application of $\mathrm{BS}$ as a dichotomise measure can only provide information on presence of hypermobile joint $[4,5,31]$. Furthermore, there are currently no gold standard method for GJH diagnosis to allow measurements of sensitivity and specificity of the BS as a diagnostic tool and therefore it limits BS application beyond an initial screening tool [30]. As the overarching aim of our systematic review was to collect evidence on the outcome measures related to symptomatic hypermobility and therefore determining the relationship between degree of hypermobility and these outcomes was outside the scope of our review. As far as we are aware there are no studies that have correlated grade of lower limb hypermobility to the degree of these domains in children and certainly this is a valid question worth exploration in future studies.

\section{Conclusions}

An agreed set of core outcome measures for children with GJH and associated symptoms is warranted. More precisely defined diagnostic criteria for children with 
hypermobility related disorders, in conjunction with standardised reporting of the effectiveness of interventions using similar outcome measures in future studies will produce better quality evidence to facilitate translation into healthcare services. We recommend the development of a core set of outcome measures based around the four constructs of pain, function, quality of life and fatigue. Mixed methodology, including the views of children living with GJH and associated symptoms and their families on what is important to them, combined with expert consensus, validation of generic outcome measures in this population and development of condition specific outcome measures, would provide the ideal final core outcome set for future use.

\section{Abbreviations}

APARQ: Adolescent Physical Activity Recall Questionnaire BAP-PIQ:Bath Adolescent Pain Parent Impact; BAPQ 61: Bath Adolescent Pain questionnaire; CHAQ: Childhood Health Assessment Questionnaire; CHQ-PF50: Child Health Questionnaire; CHU9D: Child Health Utility 9D; CRO: clinician-reported outcome; hEDS: hypermobile Ehlers-Danlos Syndrome; G-HSD: Generalised Hypermobility Spectrum Disorder; GJH: Generalised joint hypermobility; Global-VAS: parent's global assessment; JHS: Joint Hypermobility Syndrome; JIA: Juvenile Idiopathic Arthritis, M-ABC2:Movement Assessment Battery for Children, 2nd Edition NRS:Numerical Rating Scale; PedsQL: Pediatric Quality of life questionnaire; PGIC: Patient's Global Impression of Change; PODCI: Pediatric Outcomes Data Collection Instrument; PRISMA: Preferred Reporting Items for Systematic Reviews and Meta-Analyses; PRO: patient- or parentreported outcome; PROSPERO: Prospective Register of Systematic Review; RCT : Randomised controlled trial; VAS: patient-reported Visual Analogue Scale; WBFPS: Wong-Baker Faces Pain Scale.

\section{Acknowledgements}

Not applicable.

\section{Authors' contributions}

MM, VP, LT and AC designed and conceptualised the systematic review. Titles, abstracts and full-text article screening was performed independently by two authors (MM and $\mathrm{AC}$ ) against the inclusion/exclusion criteria. MM and CW extracted the data; MM and DS performed the risk of bias assessment. MM, VP and LT drafted and revised the manuscript. All authors reviewed the systematic review and revised the manuscript. All authors have read and approved the final manuscript.

\section{Funding}

Muhammad Maarj is supported by University of Newcastle under a government funded position (Research Training Program fee offset scholarship).

\section{Availability of data and materials}

The datasets used and/or analysed during the current study are available from the corresponding author on reasonable request.

\section{Declarations}

Ethics approval and consent to participate Not applicable.

\section{Consent for publication}

Not applicable.

\section{Competing interests}

The authors declare that they have no competing interests.

\section{Author details}

${ }^{1}$ Narrabeen Sports Medicine Centre, Sydney Academy of Sport, Sydney, Australia. ${ }^{2}$ Department of Health Sciences, Newcastle University, Newcastle, Australia. ${ }^{3}$ Priority Research Centre Health Behaviour, Hunter Medical Research Institute HMRI, Newcastle, Australia. ${ }^{4}$ Department of Health Professions, Macquarie University, Sydney, Australia. ${ }^{5}$ Department of Physiotherapy, Monash University, Melbourne, Australia. ${ }^{6}$ Department of Health Sciences, Queen Margaret University, Scotland, UK.

Received: 18 February 2021 Accepted: 15 November 2021

Published online: 29 November 2021

\section{References}

1. Kirk J, Ansell B, Bywaters E. The hypermobility syndrome. Musculoskeletal complaints associated with generalized joint hypermobility. Ann Rheumatic Dis. 1967;26(5):419.

2. Sperotto F, Balzarin M, Parolin M, Monteforte N, Vittadello F, Zulian F. Joint hypermobility, growing pain and obesity are mutually exclusive as causes of musculoskeletal pain in schoolchildren. Clin Exp Rheumatol. 2014;32(1):131-6.

3. Remvig L, Kümmel C, Kristensen JH, Boas G, Juul-Kristensen B. Prevalence of generalized joint hypermobility, arthralgia and motor competence in 10-year-old school children. Int Musculoskeletal Med. 2011;33(4):137-45.

4. Castori M, Tinkle B, Levy H, Grahame R, Malfait F, Hakim A, editors. A framework for the classification of joint hypermobility and related conditions. Am J Med Genet C Semin Med Genet; 2017: Wiley Online Library.

5. Malfait F, Francomano C, Byers P, Belmont J, Berglund B, Black J, et al. The 2017 international classification of the Ehlers-Danlos syndromes. Am J Med Genet C Semin Med Genet. 2017;175(1):8-26.

6. Tofts $L J$, Elliott EJ, Munns C, Pacey V, Sillence DO. The differential diagnosis of children with joint hypermobility: a review of the literature. Pediatric Rheumatology. 2009;7(1):1.

7. Tinkle BT, Bird HA, Grahame R, Lavallee M, Levy HP, Sillence D. The lack of clinical distinction between the hypermobility type of Ehlers-Danlos syndrome and the joint hypermobility syndrome (aka hypermobility syndrome). Am J Med Genet Part A. 2009;149(11):2368-70.

8. Grahame R. Hypermobility: an important but often neglected area within rheumatology. Nat Clin Pract Rheumatol. 2008;4(10):522-4.

9. Voermans NC, Knoop H, Bleijenberg G, van Engelen BG. Fatigue is associated with muscle weakness in Ehlers-Danlos syndrome: an explorative study. Physiotherapy. 2011;97(2):170-4.

10. Hanewinkel-van Kleef YB, Helders PJ, Takken T, Engelbert RH. Motor performance in children with generalized hypermobility: the influence of muscle strength and exercise capacity. Pediatr Phys Ther. 2009;21(2):194-200.

11. Fatoye F, Palmer S, Macmillan F, Rowe P, van der Linden M. Pain intensity and quality of life perception in children with hypermobility syndrome. Rheumatol Int. 2012;32(5):1277-84.

12. Mu W, Muriello M, Clemens JL, Wang Y, Smith CH, Tran PT, et al. Factors affecting quality of life in children and adolescents with hypermobile Ehlers-Danlos syndrome/hypermobility spectrum disorders. Am J Med Genet Part A. 2019;179(4):561-9.

13. Armon K. Musculoskeletal pain and hypermobility in children and young people: is it benign joint hypermobility syndrome? Dis Child. 2015;100(1):2-3.

14. Pacey $V$, Tofts $L$, Adams R, Nicholson L. Factors affecting change in children with joint hypermobility syndrome: Results of a prospective longitudinal study. Int Med J. 2015;45:9.

15. Engelbert RHH, Kooijmans FT, van Riet AM, Feitsma TM, Uiterwaal CS, Helders PJ. The relationship between generalized joint hypermobility and motor development. Pediatr Phys Ther. 2005;17(4):258-63.

16. Fatoye F, Palmer S, Macmillan F, Rowe P, van der Linden M. Proprioception and muscle torque deficits in children with hypermobility syndrome. Rheumatology (Oxford). 2009:48(2):152-7.

17. Schubert-Hjalmarsson E, Öhman A, Kyllerman M, Beckung E. Pain, balance, activity, and participation in children with hypermobility syndrome. Pediatr Phys Ther. 2012;24(4):339-44.

18. Scheper MC, Juul-Kristensen B, Rombaut L, Rameckers EA, Verbunt J, Engelbert RH. Disability in Adolescents and Adults Diagnosed With 
Hypermobility-Related Disorders: A Meta-Analysis. Arch Physical Med Rehabil. 2016;97(12):2174-87.

19. Zekry OA, Ahmed MA, Elwahid HAEA. The impact of fatigue on health related quality of life in adolescents with benign joint hypermobility syndrome. Egypt Rheumatol. 2013;35(2):77-85.

20. Baeza-Velasco C, Gély-Nargeot M, Vilarrasa AB, Bravo J. Joint hypermobility syndrome: problems that require psychological intervention. Rheumatol Int. 2011;31(9):1131-6.

21. Rome K, Ashford RL, Evans A. Non-surgical interventions for paediatric pes planus. Cochrane Database of systematic reviews. 2010(7).

22. Scheper MC, Engelbert RHH, Rameckers EAA, Verbunt J, Remvig L, JuulKristensen B. Children with generalised joint hypermobility and musculoskeletal complaints: state of the art on diagnostics, clinical characteristics, and treatment. BioMed Res Int. 2013;2013:121054-

23. Peterson B, Coda A, Pacey V, Hawke F. Physical and mechanical therapies for lower limb symptoms in children with Hypermobility Spectrum Disorder and Hypermobile Ehlers-Danlos Syndrome: a systematic review. J Foot Ankle Res. 2018;11:59.

24. Engelbert RHH, Scheper M, Rameckers E, Verbunt J, Remvig L, Juul-Kristensen B. Children with generalized joint hypermobility and musculoskeletal complaints: State of the art on diagnostics, clinical characteristics and treatment. Annals of the Rheumatic Diseases. 2013;72.

25. Smith TO, Bacon H, Jerman E, Easton V, Armon K, Poland F, et al. Physiotherapy and occupational therapy interventions for people with benign joint hypermobility syndrome: a systematic review of clinical trials. Disabil Rehabil. 2014;36(10):797-803.

26. Gargon E, Gorst SL, Williamson PR. Choosing important health outcomes for comparative effectiveness research: 5 th annual update to a systematic review of core outcome sets for research. PloS one. 2019;14(12):e0225980.

27. Moher D, Hopewell S, Schulz KF, Montori V, Gøtzsche PC, Devereaux PJ, et al. CONSORT 2010 Explanation and Elaboration: updated guidelines for reporting parallel group randomised trials. BMJ. 2010;340:c869.

28. Beighton P, Solomon L, Soskolne C. Articular mobility in an African population. Ann Rheumatic Dis. 1973;32(5):413.

29. Carter C, Wilkinson J. Persistent joint laxity and congenital dislocation of the hip. J Bone Joint Surg Brit Vol. 1964;46(1):40-5.

30. Malek S, Reinhold EJ, Pearce GS. The Beighton Score as a measure of generalised joint hypermobility. Rheumatol Int. 2021:1-10.

31. Juul-Kristensen B, Schmedling K, Rombaut L, Lund H, Engelbert RH, editors. Measurement properties of clinical assessment methods for classifying generalized joint hypermobility — a systematic review. Am J Med Genet Part C: Seminars Med Genet 2017: Wiley Online Library.

32. Smits-Engelsman B, Klerks M, Kirby A. Beighton score: a valid measure for generalized hypermobility in children. J Pediatr. 2011;158(1):119-23, 23.e1-4.

33. Singh H, McKay M, Baldwin J, Nicholson L, Chan C, Burns J, et al. Beighton scores and cut-offs across the lifespan: cross-sectional study of an Australian population. Rheumatology. 2017;56(11):1857-64.

34. Jansson A, Saartok T, Werner S, Renström P. General joint laxity in 1845 Swedish school children of different ages: age-and gender-specific distributions. Acta Paediatrica. 2004:93(9):1202-6.

35. Downs SH, Black N. The feasibility of creating a checklist for the assessment of the methodological quality both of randomised and nonrandomised studies of health care interventions. J Epidemiol Commun Health. 1998:52(6):377-84.

36. Deeks JJ, Dinnes J, D'Amico R, Sowden AJ, Sakarovitch C, Song F, et al. Evaluating non-randomised intervention studies. Health Technol Assess. 2003;7(27):iii-X, 1-173.

37. Theis J, Gerdhem P, Abbott A. Quality of life outcomes in surgically treated adult scoliosis patients: a systematic review. Eur Spine J. 2015;24(7):1343-55

38. Chudyk AM, Jutai JW, Petrella RJ, Speechley M. Systematic review of hip fracture rehabilitation practices in the elderly. Arch Physical Med Rehabil. 2009;90(2):246-62.

39. Bale P, Easton V, Bacon H, Jerman E, Watts L, Barton G, et al. The effectiveness of a multidisciplinary intervention strategy for the treatment of symptomatic joint hypermobility in childhood: a randomised, single Centre parallel group trial (The Bendy Study). Pediatr Rheumatol Online J. 2019;17(1):2.
40. Hsieh RL, Peng HL, Lee WC. Short-term effects of customized arch support insoles on symptomatic flexible flatfoot in children: A randomized controlled trial. Medicine (Baltimore). 2018;97(20):e10655.

41. Kemp S, Roberts I, Gamble C, Wilkinson S, Davidson JE, Baildam EM, et al. A randomized comparative trial of generalized vs targeted physiotherapy in the management of childhood hypermobility. Rheumatology (Oxford). 2010;49(2):315-25.

42. Pacey V, Tofts L, Adams RD, Munns CF, Nicholson LL. Exercise in children with joint hypermobility syndrome and knee pain: a randomised controlled trial comparing exercise into hypermobile versus neutral knee extension. Pediatr Rheumatol Online J. 2013;11(1):30.

43. Revivo G, Amstutz DK, Gagnon CM, McCormick ZL. Interdisciplinary Pain Management Improves Pain and Function in Pediatric Patients with Chronic Pain Associated with Joint Hypermobility Syndrome. PM R. 2019;11(2):150-7.

44. Scheper MC, Nicholson LL, Adams RD, Tofts L, Pacey V. The natural history of children with joint hypermobility syndrome and Ehlers-Danlos hypermobility type: a longitudinal cohort study. Rheumatology (Oxford). 2017;56(12):2073-83.

45. Shields B, Palermo T, Powers J, Grewe S, Smith G. Predictors of a child's ability to use a visual analogue scale. Child. 2003;29(4):281-90.

46. Crossley KM, Bennell KL, Cowan SM, Green S. Analysis of outcome measures for persons with patellofemoral pain: which are reliable and valid? Arch Physical Med Rehab. 2004;85(5):815-22.

47. Wong DL, Baker CM. Pain in children: comparison of assessment scales. Pediatr Nurs. 1988;14(1):9-17.

48. Garra G, Singer AJ, Taira BR, Chohan J, Cardoz H, Chisena E, et al. Validation of the Wong-Baker FACES pain rating scale in pediatric emergency department patients. Acad Emerg Med. 2010;17(1):50-4.

49. Filocamo G, Davi S, Pistorio A, Bertamino M, Ruperto N, Lattanzi B, et al. Evaluation of 21-numbered circle and 10-centimeter horizontal line visual analog scales for physician and parent subjective ratings in juvenile idiopathic arthritis. J Rheumatol. 2010;37(7):1534-41.

50. Nugent J, Ruperto N, Grainger J, Machado C, Sawhney S, Baildam E, et al. The British version of the childhood health assessment questionnaire $(\mathrm{CHAQ})$ and the child health questionnaire (CHQ). Clin Experiment Rheumatol. 2001;19(4; SUPP/23):S163-S7.

51. Hébert L, Maltais DB, Lepage C, Saulnier J, Crête M, Perron M. Isometric Muscle Strength in Youth Assessed by Hand-held Dynamometry: A Feasibility, Reliability, and Validity Study A Feasibility, Reliability, and Validity Study. Pediatric Physical Therapy. 2011;23(3):289-99.

52. Schoemaker MM, Niemeijer AS, Flapper BC, SMITS-ENGEL SMAN BC Validity and reliability of the movement assessment battery for children-2 checklist for children with and without motor impairments. Dev Med Child Neurol. 2012;54(4):368-75.

53. Hsieh R-L, Lin MI, Huang HY, Lee WC. The relationship between the Pediatric Outcomes Data Collec-tion Instrument and functional impairment in developmentally delayed Chinese children and their parents' health: implications for child and family-centered medicine. Int J Person Centered Med. 2011;1(3).

54. Daltroy LH, Liang MH, Fossel AH, Goldberg MJ. The POSNA pediatric musculoskeletal functional health questionnaire: report on reliability, validity, and sensitivity to change. Pediatric Outcomes Instrument Development Group. Pediatric Orthopaedic Society of North America. J Pediatr Orthop. 1998;18(5):561-71.

55. Lelieveld OT, Takken T, van der Net J, van Weert E. Validity of the 6-minute walking test in juvenile idiopathic arthritis. Arthritis Rheum. 2005:53(2):304-7.

56. Utter AC, Robertson RJ, Nieman DC, Kang J. Children's OMNI scale of perceived exertion: walking/running evaluation. Med Sci Sports Exercise. 2002;34(1):139-44.

57. Stevens K. Developing a descriptive system for a new preference-based measure of health-related quality of life for children. Quality Life Res. 2009;18(8):1105-13.

58. Waters E, Salmon L, Wake M, Hesketh K, Wright M. The Child Health Questionnaire in Australia: reliability, validity and population means. Aust New Zealand J Public Health. 2000;24(2):207-10.

59. Farrar JT, Young Jr JP, LaMoreaux L, Werth JL, Poole RM. Clinical importance of changes in chronic pain intensity measured on an 11-point numerical pain rating scale. Pain. 2001;94(2):149-58. 
60. Scott W, McCracken LM. Patients'impression of change following treatment for chronic pain: global, specific, a single dimension, or many? J Pain. 2015;16(6):518-26.

61. American Journal of Medical Genetics Part C: Seminars in Medical Genetics;Chan LF, Chow SM, Lo SK. Preliminary validation of the Chinese version of the Pediatric Quality of Life Inventory. Int J Rehabil Res. 2005;28(3):219-27.

62. Varni JW, Seid M, Smith Knight T, Burwinkle T, Brown J, Szer IS. The Peds $L^{\mathrm{TM}}$ in pediatric rheumatology: reliability, validity, and responsiveness of the Pediatric Quality of Life Inventory ${ }^{\mathrm{TM}}$ Generic Core Scales and Rheumatology Module. Arthritis Rheumatism. 2002;46(3):714-25.

63. Bilsbury CD, Richman A. A staging approach to measuring patient-centred subjective outcomes. Acta Psychiatrica Scand. 2002;106:5-40.

64. Kumar B, Lenert P. Joint hypermobility syndrome: recognizing a commonly overlooked cause of chronic pain. Am J Med. 2017;130(6):640-7.

65. Engelbert RHH, Juul-Kristensen B, Pacey V, de Wandele I, Smeenk S, Woinarosky N, et al. The evidence-based rationale for physical therapy treatment of children, adolescents, and adults diagnosed with joint hypermobility syndrome/hypermobile Ehlers Danlos syndrome. Am J Med Genet C Semin Med Genet. 2017:175(1):158-67.

66. Brinker MR, O'Connor DP. Stakeholders in outcome measures: review from a clinical perspective. Clin Orthopaedics Related Res. 2013;471(11):3426-36.

67. Fayers P, Machin D. Item response theory and differential item functioning. Quality of Life the Assessment, Analysis and Interpretation of PatientReported Outcomes, 2nd ed Chichester: Wiley. 2007:161-88.

68. Morgan EM, Riebschleger MP, Horonjeff J, Consolaro A, Munro JE, Thornhill S, et al. Evidence for updating the core domain set of outcome measures for juvenile idiopathic arthritis: report from a special interest group at OMERACT 2016. J Rheumatol. 2017;44(12):1884-8.

69. Morgan EM, Munro JE, Horonjeff J, Horgan B, Shea B, Feldman BM, et al. Establishing an updated core domain set for studies in juvenile idiopathic arthritis: a report from the OMERACT 2018 JIA Workshop. J Rheumatol. 2019;46(8):1006-13.

70. Castori M, Celletti C, Camerota F, Grammatico P. Chronic fatigue syndrome is commonly diagnosed in patients with Ehlers-Danlos syndrome hypermobility type/joint hypermobility syndrome. Clin Experiment Rheumatol-Incl Supplements. 2011;29(3):597.

71. Castori M, Sperduti I, Celletti C, Camerota F, Grammatico P. Symptom and joint mobility progression in the joint hypermobility syndrome (Ehlers-Danlos syndrome, hypermobility type). Clin Exp Rheumatol. 2011;29(6):998-1005.

72. Di Mattia F, Fary R, Murray KJ, Howie E, Smith A, Morris S. Two subtypes of symptomatic joint hypermobility: a descriptive study using latent class analysis. Arch Dis Childhood. 2019;104(11):1099-101.

73. O'Connor SR, Tully MA, Ryan B, Bradley JM, Baxter GD, McDonough SM. Failure of a numerical quality assessment scale to identify potential risk of bias in a systematic review: a comparison study. BMC Res Notes. 2015;8(1):224

74. Higgins JP. green S. Cochrane handbook for systematic reviews of interventions version. 2011;5(0):3.

75. Pacey V, Tofts L, Adams RD, Munns CF, Nicholson LL. Quality of life prediction in children with joint hypermobility syndrome. J Paediatrics Child Health. 2015;51(7):689-95.

\section{Publisher's Note}

Springer Nature remains neutral with regard to jurisdictional claims in published maps and institutional affiliations.

Ready to submit your research? Choose BMC and benefit from:

- fast, convenient online submission

- thorough peer review by experienced researchers in your field

- rapid publication on acceptance

- support for research data, including large and complex data types

- gold Open Access which fosters wider collaboration and increased citations

- maximum visibility for your research: over $100 \mathrm{M}$ website views per year

At BMC, research is always in progress.

Learn more biomedcentral.com/submissions 